\title{
Applying the ICF-CY in visually impaired rehabilitation: a case report in China
}

\author{
Bingbing Li, Yang Yu, Jianmin Hu \\ Department of Ophthalmology, The Second Affiliated Hospital of Fujian Medical University, Fujian Province University Engineering Research \\ Center of Assistive Technology for Visual Impairment, Quanzhou, China \\ Correspondence to: Jianmin Hu. Doctoral Supervisor, Professor, Department of Ophthalmology, The Second Affiliated Hospital of Fujian Medical \\ University, Fujian Province University Engineering Research Center of Assistive Technology for Visual Impairment, No. 34 Zhongshanbei Road, \\ Licheng District, Quanzhou, China. Email: doctorhjm@163.com.
}

\begin{abstract}
The model provided by the International Classification of Functioning, Disability and Health (ICF) is used to describe, classify and measure function in both health care practice and research. ICF for Children and Youth (ICF-CY) has not been applied to the rehabilitation of patients with visual impairment. The purpose of this case report is to describe the process of evaluation and treatment of low vision patients based on ICF-CY models. The patient was a 9-year-old girl. The diagnosis included retinopathy of prematurity (ROP) (OU), secondary glaucoma after iridectomy (OD), keratoleukoma (OD), retinal detachment (OS), strabismus postoperation (OU), nystagmus. The patient complained of being unable to see clearly, of fear of falling down stairs and of being unable to study normally. The ICF-CY framework is used to assess the overall function and disability of this girl. We provided visual devices and instruction to improve the body structure and function, mobility restriction and environmental factors associated with the cognitive function of patients. The results showed that with the help of devices and the guidance of her parents, friends and health professionals, the patient's cognitive function, learning ability and social ability were significantly improved and her psychological conditions had also been improved. As a result, using the ICF-CY model to evaluate and intervene in patients is helpful for accurately dealing with different functional problems and their correlations.
\end{abstract}

Keywords: International Classification of Functioning, Disability and Health for Children and Youth (ICF-CY); retinopathy of prematurity (ROP); rehabilitation of visual impairment; case report

Submitted Feb 05, 2020. Accepted for publication Aug 07, 2020.

doi: $10.21037 / \mathrm{apm}-20-312$

View this article at: http://dx.doi.org/10.21037/apm-20-312

\section{Introduction}

Retinopathy of prematurity (ROP) is a kind of retinal vascular system that proliferates under the stimulation of hypoxia and other factors in premature and lowweight infants. In severe cases, ROP can lead to retinal detachment, which can severely affect the development of vision and eye in children, as well as normal language, cognitive and social development. In recent years, with the improvement of medical condition and level, the survival rate of premature infants has been increasing, which has a major cause of blindness in children in the developing and developed world (1). Therefore, the early treatment of ROP is very important, once missed the best opportunity for treatment will lead to low vision. Having visual impairment significantly affects an individual's daily functioning and quality of life (2). Visually impaired children must be treated by a multidisciplinary rehabilitation team. The ultimate goal of rehabilitation is to enhance low vision patients' participation in society. Under the ophthalmology clinical rehabilitation of visual impairment, appropriate and accurate tools for measuring disability in children are essential.

The International Classification of Functioning, 


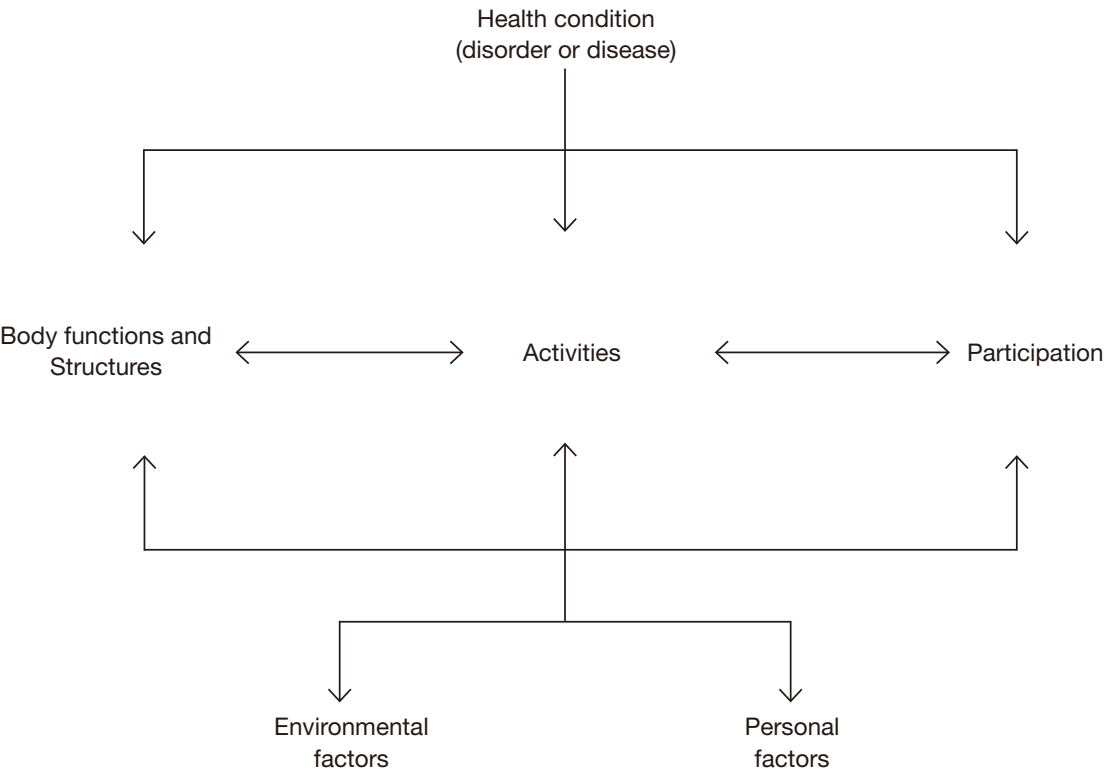

Figure 1 ICF conceptual framework. ICF, International Classification of Functioning, Disability and Health.

Disability and Health (ICF) was the achievement of an effort in 1993 involving 1,800 experts from 65 countries. And it was eventually accepted as the international standard for describing and measuring health and disability in 2001 (Figure 1). In October 2007, the World Health Organization published an adaptation of the ICF especially designed for children and youth, based on an ecological model that conceptualizes a child's adaptation as a function of ongoing interactions between the child (and his or her characteristics) and the environment over time $(3,4)$. The ICF for Children and Youth (ICF-CY) is a classification tool derived from the ICF for children and youth from birth to the age of 18 , and is a structured measure: its codes consist of a letter (b, s, d, and e) followed by 1 or more numbers. The letter refers to the domain and the numbers to the level of specification of the domain (5). Thus, the ICF-CY is a classification that describes the functioning of children and youth in detail from different perspectives, such as body functions, anatomical characteristics, activities, and participation. Furthermore, ICF-CY takes into account the social aspects of human functioning and does not view disability as merely a "medical" or "biological" disorder. It was served as a framework for rethinking evaluation, goals and interventions. It aims to help clinicians, educators, public policy makers, family members, consumers and researchers identify the health and education needs of developing children. The purpose of this single case study is to use ICF-CY as a comprehensive assessment tool to better understand and improve rehabilitation pathways for visually impaired children, and to illustrate the application of ICF-CY in the multidisciplinary team approach to visual rehabilitation (Figure 2). We present the following case in accordance with the CARE reporting checklist (available at http://dx.doi.org/10.21037/apm-20-312).

\section{Case presentation}

The subject is a 9-year-old girl, came to the hospital for treatment of "premature infant with retinopathy of prematurity detected by fundus screening for 9 years" in 2018. The diagnosis included "ROP (OU), secondary glaucoma after iridectomy (OD), keratoleukoma (OD), retinal detachment (OS), strabismus postoperation (OU), nystagmus". There was nothing special about the family history of the patient, and genetic testing didn't suggest abnormalities. The patient complained of being unable to see clearly, of fear of falling down stairs and of being unable to study normally. The stated goal of the patient and her parents was to improve cognitive function.

On admission, the patient had no light perception in her right eye, visual acuity of her left eye is 20/1,000, nystagmus $(\mathrm{OU})$. For her right eye, keratoleukoma, disappearance of anterior chamber, patency of the incision around the superior iris, posterior synechia of the iris, the posterior 


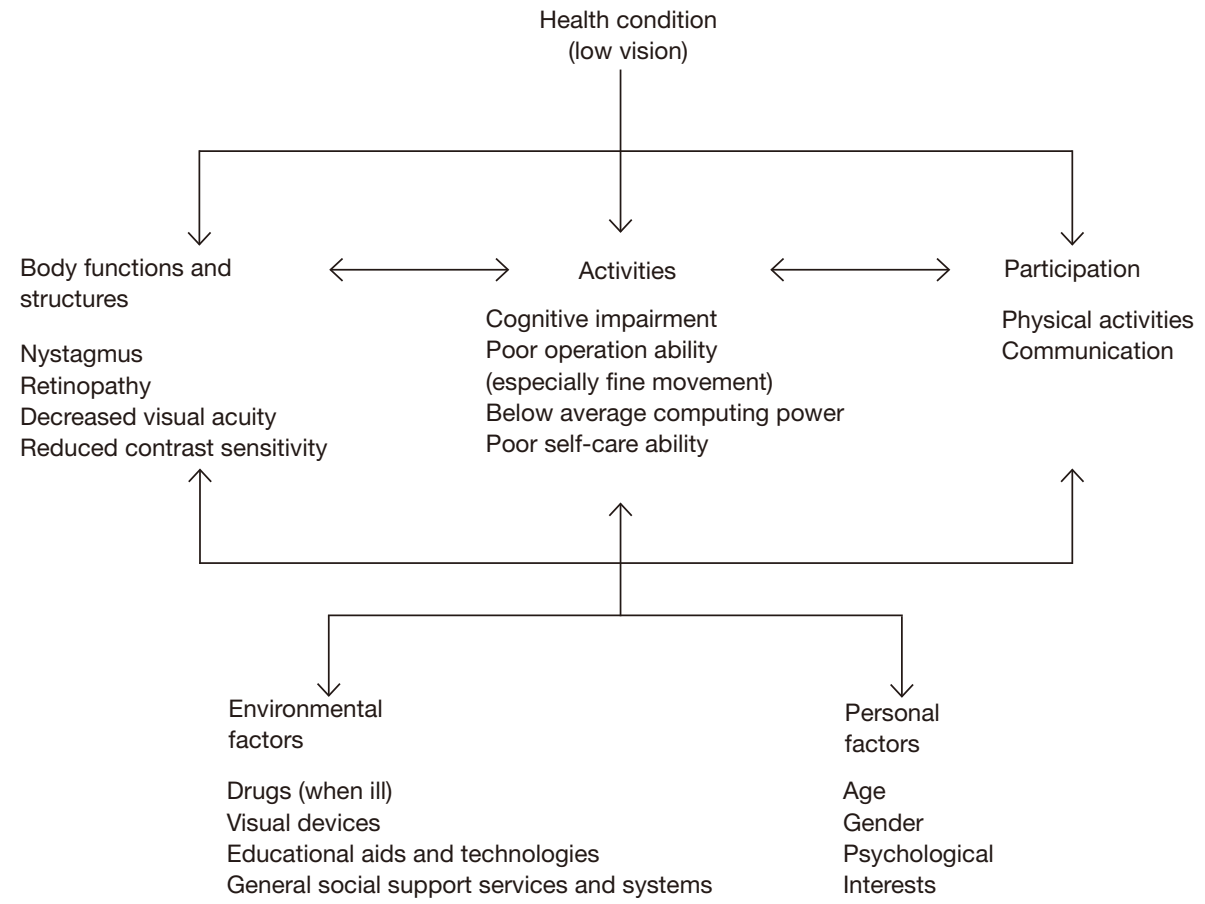

Figure 2 ICF-CY conceptual framework. ICF-CY, International Classification of Functioning, Disability and Health for Children and Youth.

segment was invisible, and the b-mode ultrasonography revealed atrophy of the right eye. There was no obvious abnormality in the anterior segment of the left eye. The fundus of the left eye was lined with retinal folds from the temporal disc to the peripheral retina. In the left eye, there were $3^{\circ}$ (diameter) in the temporal center of the visual field, macular tilt in the optic nerve fiber layer. An examination plan was made, which included the low vision quality of life questionnaire (LVQOL), the Montreal cognitive assessment scale (MOCA), Achenbach child behavior scale (CBCL), and the infant-junior middle school life ability scale (SM). The behavior, cognition and psychology of the patient were evaluated in many aspects, and the patient was accepted the comprehensive rehabilitation for visual impairment. The results of the above-mentioned scales suggest that the patient had cognitive dysfunction, poor performance (especially fine motion), learning ability lower than average, and social withdrawal. Therefore, we provided her with a visual device and instructed her how to use.

The patient was referred to our clinic for a comprehensive rehabilitation for the visually impaired. In view of her age and functional characteristics, the objective of the rehabilitation for the visually impaired agreed with her family was to enhance function. Consistent with these views, the patient use ICF-CY classification codes integrated into the visual rehabilitation format. An expert multidisciplinary team, including ophthalmologists, pediatricians, psychologists, educators, and computerassistive technology specialists (each in their own field), was involved to identify a selected set of codes for our patient.

The first step is to conduct interviews to identify problems encountered by the child and her families. After that, tests were performed to examine and select each ICF-CY category. The second step is to set an overall and periodic goal for rehabilitation based on the wishes of the patient and her parents. According to the rehabilitation goals, cognitive ability was set as a major prognostic indicator. The third step is to identify treatment that correspond to the periodic goals, using an ICF-CY-based rehabilitation for the visually impaired (Figure 3). The MOCA was used to assess cognitive impairment; the CBCL was used to assess activity, social interaction and school performance; and the SM was used to assess various life abilities such as independent living, sports, communication, participation in activities; the LVQOL was used to evaluate patient's life-related quality. During the interview, parents' perceptions of their child's skill and activity performance were assessed. To describe the current functional status 


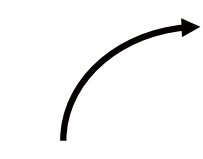

Evaluation

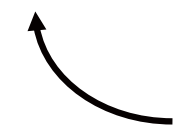

Assessment

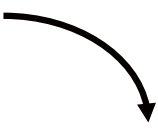

Assignment

Intervention

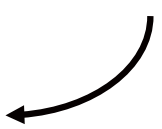

Figure 3 Rehabilitation cycle.

of the patient, 93 ICF-CY categories were selected: 15 functional categories, 6 structural categories, 57 activity and participation categories, and 15 environmental factors.

The patient was coded using the ICF-CY questionnaire for ages 7 to 12 years. Her functioning was considered in her primary environments (e.g., home, school, community) (Figure 4). Each ICF-CY code was completed with the relative qualifier. Qualifiers denoted the extent or magnitude of the problem for a given code (5). The patient's problems were identified primarily in the activities and participation section, these included self-care functions (dressing, traveling and doing housework), learning functions (learning to read, writing, and calculating), and social functions (basic interpersonal communication, basic economic exchange, and community life). The domains of the activities and participation component were translated through the use of two qualifiers: "capacity" (defined as what the child can do) and "performance" (defined as what the child actually does) (5). Barriers in the environment included the lack of appropriate medications for visual impairment, the lack of supplies and technology for individuals' daily lives, and the lack of assistive technologies for communication and learning. The interventions in the individual rehabilitation were determined on the basis of environmental factors, including the use of devices and techniques for the daily life of the individual (e115), such as the instruction of devices, and aids and techniques for education (e1301). There was also help from parents and friends (e310, e320), guidance from health professionals (e3559) and general social support services and systems (e575) (Table 1).

The patient came to our clinic again in 2019 , and we conducted the same scales survey as 1 year ago to evaluate the treatment outcome. Confirmed with the patient's family that the patient was cooperative with the therapeutic intervention during the past year. To determine the improvement, a comparison of clinical improvement from

pretreatment to post-treatment scores was made (Table 2). The MOCA indicated that the patient's cognitive ability improved. The CBCL showed the improvement of learning and social ability, and the improvement of social ability was significant. The CBCL also suggested that the psychological status of the patient had also been significantly improved. The SM indicated that the patient's daily life skills had also been improved, and the results of the LVQOL indicated that the clinical improvement between baseline assessment and reassessment is indicative. We used the ICF-CY code to record the gradients and levels of changes in physical function or structure, activity performance or participation in social roles. The results showed that the patient's cognitive function was significantly improved with the help of devices and the guidance of their parents, friends and professionals. No adverse or unanticipated events happened in this case. The patient's rehabilitation program (Figure 5) showed changes in ICF-CY levels.

All clinical investigations were approved by the Ethical Review Committee of The Second Affiliated Hospital of Fujian Medical University (No. 2019-170), and written informed consent was obtained from the patient.

\section{Discussion}

The ICF-CY model is unique in that there is not a hierarchical organization of its domains but rather a bidirectional flow of information. This allows physical therapists to better understand the impact of a patient's impairments in relation to their daily life and facilitates prioritization of interventions to remedy these various domains. As this framework also aids in showcasing a patient's potential barriers, these too can be addressed to allow progression toward goals in a timely fashion (6).

In this case study, ICF-CY was used as an assessment tool to guide decision-making in helping a visually impaired child with ROP to improve her cognitive ability. The ICF$\mathrm{CY}$ provides this common and universal language for describing and measuring health and disability in the first two decades of life, as well as a conceptual framework to guide operationalizing constructs and framing findings in a manner that conveys meaning across disciplines (3). In addition, the involvement of families of visually impaired children expanded the range of rehabilitation interventions include the patient's environment, the functional goals of the patient and her family, and the physical abilities of the patient. The ICF-CY is a useful biopsychosocial framework to "rule in" relevant aspects of child and family 


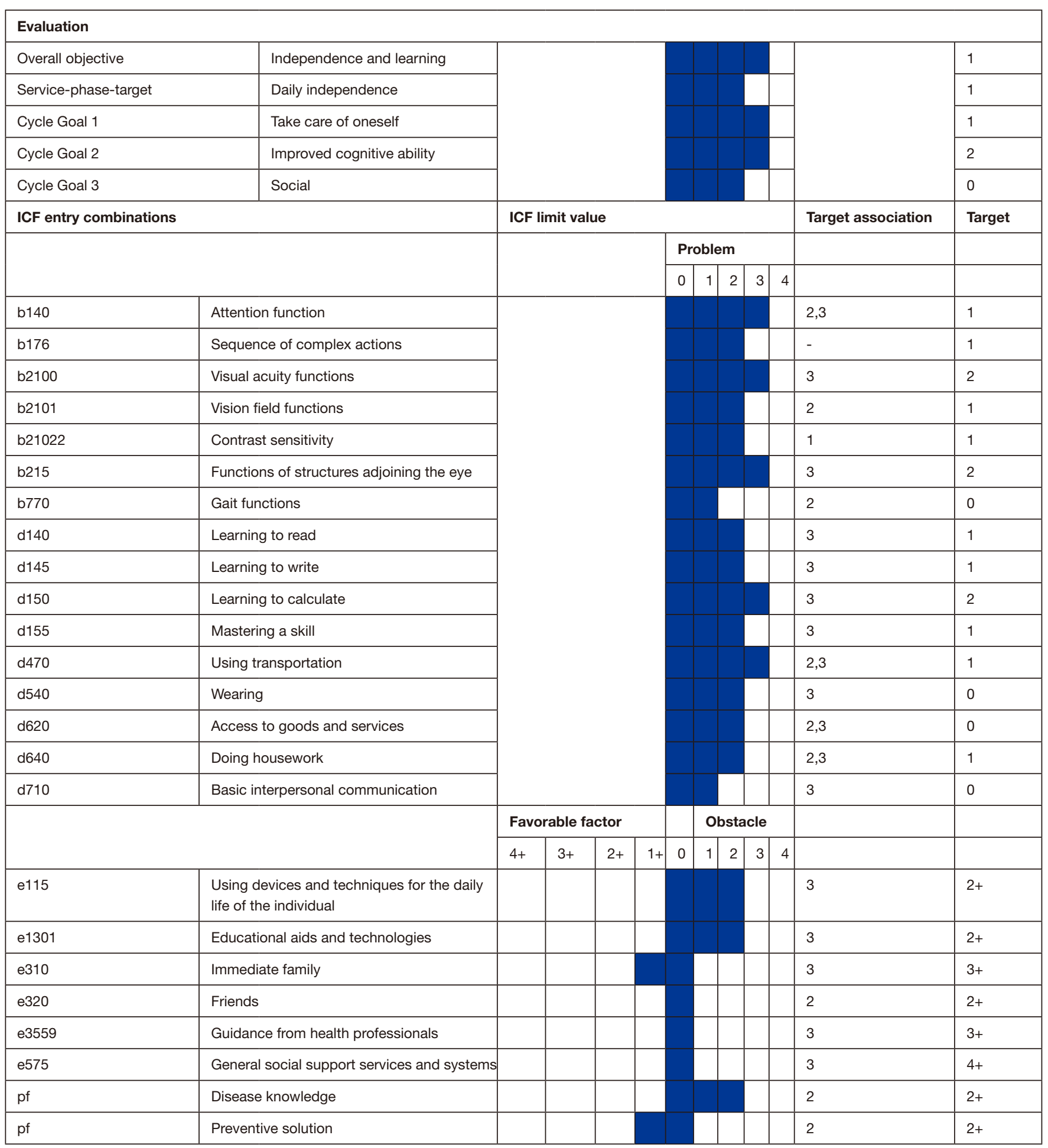

Figure 4 ICF-CY entry combinations and goal setting. In the ICF limit, body function/structure, activities and participation: $0=$ no problem, $1=$ mild problem, $2=$ moderate problem, $3=$ serious problem, $4=$ completely problem. In the related factors: $0=$ no obstacle/promotion, $1 / 1+=$ mild obstacle/promotion, 2/2+= moderate obstacle/promotion, 3/3+= serious obstacle/promotion, 4/4+= completely obstacle/promotion. ICF-CY, International Classification of Functioning, Disability and Health for Children and Youth. 


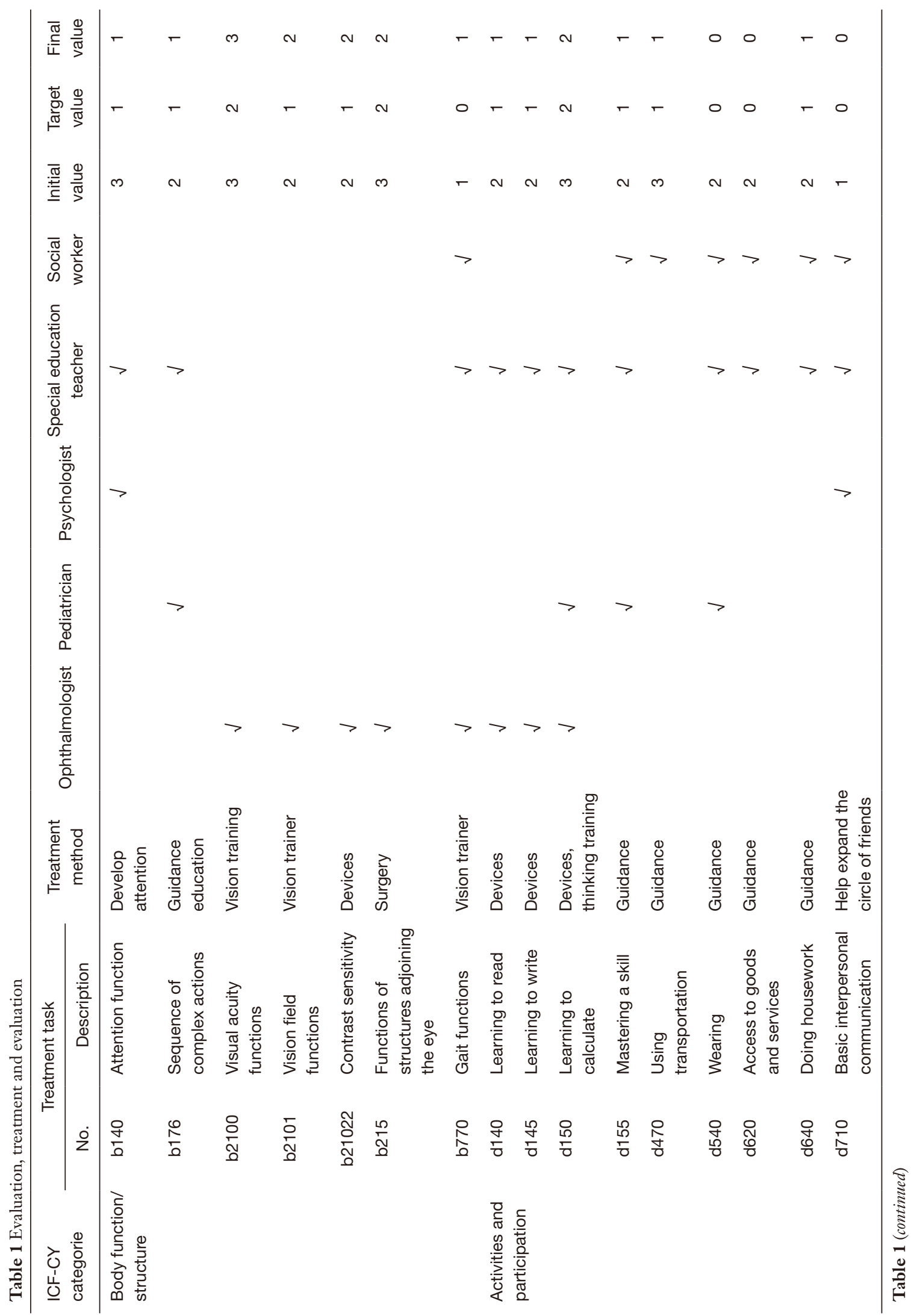




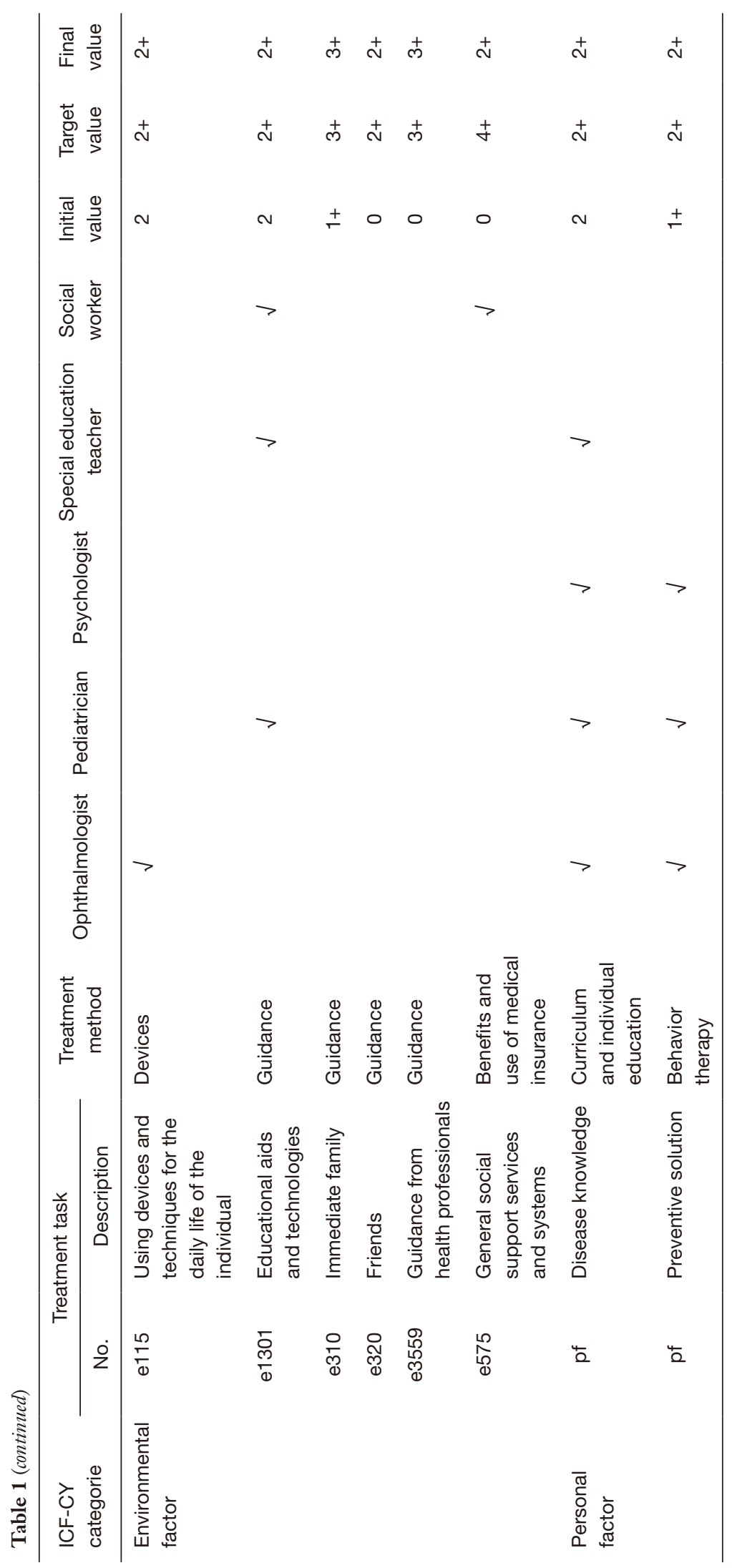


Table 2 Patient assessment score

\begin{tabular}{lccc}
\hline \multirow{2}{*}{ Scales } & \multicolumn{3}{c}{ Score } \\
\cline { 2 - 4 } & 2018 & 2019 & Maximum \\
\hline LVQOL & 55 & 73 & 120 \\
MOCA & 13 & 20 & 30 \\
CBCL & 13 & 19.4 & 30 \\
SM & 34 & 80 & 132 \\
\hline
\end{tabular}

LVQOL, low vision quality of life questionnaire; MOCA, Montreal cognitive assessment scale; CBCL, Achenbach child behavior scale; SM, the infant-junior middle school life ability scale.

issues to create a dynamic system of possible influences on outcomes (7). Certainly, one of the difficulties associated with the use of ICF-CY in clinical practice is that there are many categories, totaling more than 1,400 . In order to promote the implementation of ICF-CY, the so-called "ICF core sets" has been developed. ICF-CY has not yet developed a core language for visual impairment, so we need to further explore a conceptual framework for the functions and needs of visually impaired children. A Brief ICF Core Set for a specific condition includes a list of ICF categories, with as few categories as possible to be practical, but as many as necessary to be sufficiently comprehensive to describe-in clinical studies and possibly clinical encounters-the typical spectrum of problems in the functioning of patients with a specific condition (8). Furthermore, as an approach, it operationalizes the ICF for clinical practice and research and can serve as a reference framework and a practical tool to classify and describe patient functioning in a more time-efficient way $(9,10)$.

In our study, a set of selected codes was used to define the basic dimensions of the patient, which could serve as the core set of ICF-CY identified by the multidisciplinary team. We recognized the functional aspects of the need for evaluation and treatment of patients. In particular, the ICF-CY-based program built for our patient provides a comprehensive description of its function, targets specific rehabilitation goals, and records any changes in its function. The aim of this single case study was to explore the functional changes in all ICF-CY activity and participation levels and body structure and function in children associated with the visually impaired rehabilitation program.

The most significant findings from our study were changes in activities and participation, which were observed after interventions on environmental factors associated with visual impairment. These findings are consistent with the conceptual framework of the ICF-CY in which improvement may be maximized through the intervention on the patient's environment and the family (5). Therefore, the ICF-CY domain plays a primary role in any health intervention.

The limitation of our study is single case design. The effect of applying the ICF-CY framework in the assessment and rehabilitation of visually impaired children is obvious, but case accumulation and systematic efficacy evaluation are still needed. In addition, this study is the first attempt to apply ICF-CY to the assessment and rehabilitation of visually impaired children in China, so we lack enough experience. The patient was referred from another province and the previous treatment data were not kept completely. We will try our best to avoid this deficiency in our future work. On the other hand, the patient is a 9-year-old child who is still growing and developing, we only conducted a 1-year follow-up assessment. However, her living needs and learning needs continue to grow with age, so it still needs long-term follow-up. Despite these limitations, the study is significant because after analyzing the correlation between patient's functional problems, different interventions are gradually proposed according to specific goals, instead of the traditional general interventions for patients with visual impairment. Furthermore, our findings encourage us to identify an ICF-CY core set for visually impaired children. The results of this single case study are preliminary and need to be tested in a large trial of visually impaired children. Once its versatility is verified, the rehabilitation model could be extended to children with visual impairment across the country in the future to help them improve their cognitive abilities.

\section{Conclusions}

In this study, the ICF-CY core set was used to identify functional issues in patient with visual impairment, and to 


\begin{tabular}{|c|c|c|c|c|c|c|c|c|c|c|c|c|c|c|c|c|c|c|c|c|c|c|}
\hline \multicolumn{13}{|c|}{ Assessment } & \multicolumn{10}{|c|}{ Evaluation } \\
\hline \multicolumn{2}{|c|}{ Overall objective: independence and learning } & & & & & & & & & & & 1 & & & & & \multicolumn{5}{|c|}{ Not yet rated } & \\
\hline \multicolumn{2}{|c|}{ Cycle Goal 1: take care of oneself } & & & & & & & & & & & 1 & & & & & & & & & & $\sqrt{ }$ \\
\hline \multicolumn{2}{|c|}{ Cycle Goal 3: social } & & & & & & & & & & & 0 & & & & & & & & & & $\sqrt{ }$ \\
\hline \multicolumn{2}{|c|}{ ICF entry combinations } & \multicolumn{9}{|c|}{ ICF limit value } & $\begin{array}{l}\text { Target } \\
\text { association }\end{array}$ & Target & \multicolumn{9}{|c|}{ ICF limit value } & $\begin{array}{l}\text { Reach } \\
\text { the goal }\end{array}$ \\
\hline b2100 & Visual acuity functions & & & & & & & & & & 3 & 2 & & & & & & & & & & - \\
\hline b2101 & Vision field functions & & & & & & & & & & 2 & 1 & & & & & & & & & & - \\
\hline b21022 & Contrast sensitivity & & & & & & & & & & 1 & 1 & & & & & & & & & & - \\
\hline b215 & $\begin{array}{l}\text { Functions of structures adjoining } \\
\text { the eye }\end{array}$ & & & & & & & & & & 3 & 2 & & & & & & & & & & $\sqrt{ }$ \\
\hline b770 & Gait functions & & & & & & & & & & 2 & 0 & & & & & & & & & & - \\
\hline d140 & Learning to read & & & & & & & & & & 3 & 1 & & & & & & & & & & $\sqrt{ }$ \\
\hline d145 & Learning to write & & & & & & & & & & 3 & 1 & & & & & & & & & & $\sqrt{ }$ \\
\hline d150 & Learning to calculate & & & & & & & & & & 3 & 2 & & & & & & & & & & $\sqrt{ }$ \\
\hline & & $4+$ & $3+$ & $2+$ & $1+$ & 0 & 1 & 2 & 3 & 4 & & & $4+$ & $3+$ & $2+$ & $1+$ & 0 & 1 & 2 & 3 & 4 & \\
\hline e115 & $\begin{array}{l}\text { Using devices and techniques for } \\
\text { the daily life of the individual }\end{array}$ & & & & & & & & & & 3 & $2+$ & & & & & & & & & & $\sqrt{ }$ \\
\hline e1301 & Educational aids and technologies & & & & & & & & & & 3 & $2+$ & & & & & & & & & & $\sqrt{ }$ \\
\hline e310 & Immediate family & & & & & & & & & & 3 & $3+$ & & & & & & & & & & $\sqrt{ }$ \\
\hline e320 & Friends & & & & & & & & & & 2 & $2+$ & & & & & & & & & & $\sqrt{ }$ \\
\hline e3559 & Guidance from health professionals & & & & & & & & & & 3 & $3+$ & & & & & & & & & & $\sqrt{ }$ \\
\hline e575 & $\begin{array}{l}\text { General social support services } \\
\text { and systems }\end{array}$ & & & & & & & & & & 3 & $4+$ & & & & & & & & & & $\sqrt{ }$ \\
\hline $\mathrm{pf}$ & Disease knowledge & & & & & & & & & & 2 & $2+$ & & & & & & & & & & $\sqrt{ }$ \\
\hline pf & Preventive solution & & & & & & & & & & 2 & $2+$ & & & & & & & & & & $\sqrt{ }$ \\
\hline
\end{tabular}

Figure 5 Evaluation report 1-year after rehabilitation training. ICF, International Classification of Functioning, Disability and Health. 
prioritize issues to apply interventions gradually. The use of the ICF-CY model enables patients to perform general functional assessments and apply appropriate goals and interventions. Using the ICF-CY model to perform these interventions on patients is conducive to accurately handling different functional problems and their correlations.

\section{Acknowledgments}

Funding: This study was funded by Science Research Foundation of Ministry of Health United Fujian Provincial Health, and Education Project for Tackling the Key Research (award number WKJ2016-2-12) and National Key R\&D Program of China Sub-Project Fund for Scholar (award number 2018YFC2002602).

\section{Footnote}

Reporting Checklist: The authors have completed the CARE reporting checklist. Available at http://dx.doi.org/10.21037/ apm-20-312

Conflicts of Interest: All authors have completed the ICMJE uniform disclosure form (available at http://dx.doi. org/10.21037/apm-20-312). The authors have no conflicts of interest to declare.

Ethical Statement: The authors are accountable for all aspects of the work in ensuring that questions related to the accuracy or integrity of any part of the work are appropriately investigated and resolved. All clinical investigations were approved by the Ethical Review Committee of The Second Affiliated Hospital of Fujian Medical University (No. 2019-170), and written informed consent was obtained from the patient. All procedures performed in studies involving human participants were in accordance with the Helsinki Declaration (as revised in 2013).

Open Access Statement: This is an Open Access article distributed in accordance with the Creative Commons Attribution-NonCommercial-NoDerivs 4.0 International
License (CC BY-NC-ND 4.0), which permits the noncommercial replication and distribution of the article with the strict proviso that no changes or edits are made and the original work is properly cited (including links to both the formal publication through the relevant DOI and the license). See: https://creativecommons.org/licenses/by-nc-nd/4.0/.

\section{References}

1. Chen J, Stahl A, Hellstrom A, et al. Current update on retinopathy of prematurity: screening and treatment. Curr Opin Pediatr 2011;23:173-8.

2. van Leeuwen $L M$, Rainey L, Kef S, et al. Investigating rehabilitation needs of visually impaired young adults according to the International Classification of Functioning, Disability and Health. Acta Ophthalmol 2015;93:642-50.

3. Lee AM. Using the ICF-CY to organise characteristics of children's functioning. Disabil Rehabil 2011;33:605-16.

4. Bronfenbrenner U, Ceci SJ. Nature-nurture reconceptualized in developmental perspective: a bioecological model. Psychol Rev 1994;101:568-86.

5. Trabacca A, Russo L, Losito L, et al. The ICF-CY perspective on the neurorehabilitation of cerebral palsy: a single case study. J Child Neurol 2012;27:183-90.

6. Wahlgren A, Palombaro K. Evidence-based physical therapy for BPPV using the International Classification of Functioning, Disability and Health model: a case report. J Geriatr Phys Ther 2012;35:200-5.

7. Ronen GM, Fayed N, Rosenbaum PL. Outcomes in pediatric neurology: a review of conceptual issues and recommendations. The 2010 Ronnie Mac Keith Lecture. Dev Med Child Neurol 2011;53:305-12.

8. Cieza A, Ewert T, Ustün TB, et al. Development of ICF Core Sets for patients with chronic conditions. J Rehabil Med 2004;(44 Suppl):9-11.

9. Simeonsson RJ. ICF-CY: a universal tool for documentation of disability. J Policy Pract Intellect Disabil 2009;6:70-2.

10. Stucki G, Grimby G. Applying the ICF in medicine. J Rehabil Med 2004;(44 Suppl):5-6.
Cite this article as: $\mathrm{Li} \mathrm{B,} \mathrm{Yu} \mathrm{Y,} \mathrm{Hu} \mathrm{J.} \mathrm{Applying} \mathrm{the} \mathrm{ICF-CY}$ in visually impaired rehabilitation: a case report in China. Ann Palliat Med 2021;10(3):3459-3468. doi: 10.21037/apm-20-312 